\title{
SEARCH FOR NEUTRINO EMISSION FROM GAMMA-RAY SOURCES WITH THE ANTARES TELESCOPE
}

\author{
C. BIGONGIARI \\ Instituto de Física Corpuscolar, \\ Consejo Superior de Investigación Cientifica and Universitat de València, \\ Edificios Investigación de Paterna, Apdo. de Correos 22085, \\ Valencia, E-46071, Spain \\ ciro.bigongiari@ific.uv.es
}

\begin{abstract}
ANTARES is the first undersea neutrino detector ever built and presently the neutrino telescope with the largest effective area operating in the Northern Hemisphere. A threedimensional array of photomultiplier tubes detects the Cherenkov light induced by the muons produced in the interaction of high energy neutrinos with the matter surrounding the detector. The detection of astronomical neutrino sources is one of the main goals of ANTARES. The search for point-like neutrino sources with the ANTARES telescope is described and the preliminary results obtained with data collected from 2007 to 2010 are shown. No cosmic neutrino source has been observed and neutrino flux upper limits have been calculated for the most promising source candidates.
\end{abstract}

Keywords: High energy neutrinos; Cosmic rays; Undersea neutrino telescope.

\section{Introduction}

Cosmic rays were firstly detected nearly one century ago and widely studied since then but their origin is still unknown. High energy gamma rays have been detected from astronomical sources. In particular astronomical objects with relativistic outflows, such Blazars and Micro-quasars, have been firmly established as sources of high energy gamma rays ${ }^{2}$. If these sources accelerate hadronic cosmic rays too they should produce also neutrinos which could travel unaffected to the Earth. The observation of point-like sources of cosmic neutrinos would provide a powerful insight into cosmic particle acceleration mechanisms.

\section{Detection Principle}

The extremely low interaction cross-section of neutrinos with matter and the low neutrino fluxes expected from astronomical sources ${ }^{3}$ require large instrumented volumes to detect a statistically significant neutrino signal in a reasonable amount of time. Charged current interactions of high energy muon-neutrinos produce muons which can travel hundreds of meters before decaying. Muon neutrinos can therefore be detected even if they interact far away from the detector and their direction can 
be reconstructed with sub-degree accuracy because muon direction closely follows that of the parent neutrino. The tracks of relativistic muons propagating in optically transparent dielectric media, like water or ice, can be reconstructed detecting the induced Cherenkov light with a three dimensional array of photo-multipliers. The large background from down-going muons due to cosmic ray interactions in the atmosphere can be reduced by placing the detector deeply underwater/under-ice and selecting only up-going muons as neutrino candidates. Up-going neutrinos produced in the interactions of cosmic rays in the atmosphere constitute an irreducible background.

\section{ANTARES Detector}

ANTARES is the first undersea neutrino detector ever built and presently the neutrino telescope with the largest effective area operating in the northern hemisphere ${ }^{4}$. Located in the Mediterranean Sea 40 kilometers offshore the France coast near Toulon, the ANTARES experiment is predominantly sensitive to neutrinos from the southern hemisphere in the $\mathrm{TeV}$ to $\mathrm{PeV}$ energy range. The Cherenkov light is detected by an array of photomultiplier tubes, each housed in a pressure resistant glass sphere ${ }^{5}$. The photo-multipliers are grouped in triplets along strings which are anchored to the sea bed at a depth of 2475 meters. ANTARES final configuration (may 2008) consists of twelve detection strings spaced approximately 60 meters. Each detector string contains up to 25 triplets, separated by 14.5 meters. The arrival time and charge of the photomultiplier signals are digitized 6 and transmitted to shore. Muon tracks are reconstructed by measuring the photon arrival times and the photomultiplier positions ${ }^{7}$.

\section{Data analysis}

The preliminary results presented here have been obtained analyzing data collected in 2007-2010 period with detector configurations containing between five and twelve detection strings. The overall live time is 813 days. Muon tracks have been reconstructed using a maximum likelihood fit of the observed photon arrival times. Tracks with bad reconstruction quality, quantified by a parameter based on the value of the likelihood function, have been rejected. The angular uncertainty obtained from the fit has been also required to be smaller than one degree. Selecting up-going tracks only 3058 candidate neutrinos have been selected out of $\sim 100$ million reconstructed tracks. See 9 for a detailed description of the analysis of data collected from 2007 to 2008 .

\section{Search for point-like sources}

The search for point-like neutrino sources has been carried on looking for an excess of events over the atmospheric neutrino background anywhere in the field of view (allsky search) and by testing the presence of a signal at the locations of 51 candidate 
sources (candidate list search). Candidate sources have been selected from catalogs of high energy gamma ray sources. The list includes also the most significant spot in the all-sky search performed by the IceCube collaboration with the 22 string detector configuration. See Table 1 for the list of candidate sources.

Table 1. Candidate source list. The source coordinates and the $90 \%$ C.L. limits on the flux intensity $\phi$ expressed in $10^{-8} \mathrm{GeV}^{-1} \mathrm{~cm}^{-2} \mathrm{~s}^{-1}$ units are shown.

\begin{tabular}{|c|c|c|c|c|c|c|c|}
\hline Source & $\alpha_{s}\left(^{\circ}\right)$ & $\delta_{s}\left({ }^{\circ}\right)$ & $\phi$ & Source & $\alpha_{s}\left(^{\circ}\right)$ & $\delta_{s}\left({ }^{\circ}\right)$ & $\phi$ \\
\hline HESS J1023-575 & 155.83 & -57.76 & 6.6 & SS 433 & -72.4 & 4.98 & 4.6 \\
\hline $3 \mathrm{C} 279$ & -165.95 & -5.79 & 0.1 & HESS J1614-518 & -116.42 & -51.82 & 2.0 \\
\hline GX 339-4 & -104.30 & -48.79 & 5.8 & RX J1713.7-3946 & -101.75 & -39.75 & 2.7 \\
\hline Cir X-1 & -129.83 & -57.17 & 5.8 & $3 \mathrm{C} 454.3$ & -16.50 & 16.15 & 5.5 \\
\hline MGRO J1908+06 & -73.1 & 6.27 & 0.1 & W28 & -89.57 & -23.34 & 3.4 \\
\hline ESO 139-G12 & -95.59 & -59.94 & 5.4 & HESS J0632+057 & 98.24 & 5.81 & 4.6 \\
\hline HESS J1356-645 & -151.0 & -64.50 & 5.1 & PKS 2155-304 & -30.28 & -30.22 & 2.7 \\
\hline PKS 0548-322 & 87.67 & -32.27 & 7.1 & HESS J1741-302 & -94.75 & -30.20 & 2.7 \\
\hline HESS J1837-069 & -80.59 & -6.95 & 8.0 & Centaurus A & -158.64 & -43.2 & 2.1 \\
\hline PKS 0454-234 & 74.27 & -23.43 & 7.0 & RX J0852.0-4622 & 133.0 & -46.37 & 1.5 \\
\hline IC22 hotspot & 75.45 & -18.15 & 7.0 & 1ES 1101-232 & 165.91 & -23.49 & 2.8 \\
\hline PKS 1454-354 & -135.64 & -35.67 & 5.0 & Vela X & 128.75 & -45.60 & 1.5 \\
\hline RGB J0152+017 & 28.17 & 1.79 & 6.3 & W51C & -69.25 & 14.19 & 3.6 \\
\hline Geminga & 98.31 & 17.1 & 7.3 & PKS 0426-380 & 67.17 & -37.93 & 1.4 \\
\hline PSR B1259-63 & -164.30 & -63.83 & 3.0 & LS 5039 & -83.44 & -14.83 & 2.7 \\
\hline PKS 2005-489 & -57.63 & -48.82 & 2.8 & W44 & -75.96 & 1.38 & 3.1 \\
\hline HESS J1616-508 & -116.3 & -50.97 & 2.7 & RCW 86 & -139.32 & -62.48 & 1.1 \\
\hline HESS J1503-582 & -133.54 & -58.74 & 2.8 & Crab & 83.63 & 22.1 & 4.1 \\
\hline HESS J1632-478 & -111.96 & -47.82 & 2.6 & HESS J1507-622 & -133.28 & -62.34 & 1.1 \\
\hline H 2356-309 & 0.22 & -30.63 & 3.9 & 1ES 0347-121 & 57.35 & -11.99 & 1.9 \\
\hline MSH 15-52 & -131.47 & -59.16 & 2.6 & VER J0648+152 & 102.20 & 15.27 & 2.8 \\
\hline Galactic Center & -93.58 & -29.1 & 3.8 & PKS 0537-441 & 84.71 & -44.8 & 1.3 \\
\hline HESS J1303-631 & -164.23 & -63.20 & 2.4 & HESS J1912+101 & -71.79 & 10.15 & 2.5 \\
\hline HESS J1834-087 & -81.31 & -8.76 & 4.3 & PKS $0235+164$ & 39.66 & 16.61 & 2.8 \\
\hline PKS $1502+106$ & -133.90 & 10.52 & 5.2 & IC443 & 94.21 & 22.51 & 2.8 \\
\hline PKS 0727-11 & 112.58 & 11.70 & 1.9 & & . & . & \\
\hline
\end{tabular}

No significant excess of events has been found at the location of any candidate source. The most promising source candidate is an unidentified gamma-ray source, HESS J1023-575, while the second one is a well-known Blazar, 3C 279. Neutrino flux upper limits, calculated assuming a $E_{\nu}^{-2}$ spectrum, are shown in table 1 . Such limits are the most stringent to date for many candidate sources at $\delta_{S}<-20^{\circ}$. No significant cluster of neutrino candidates has been found in the all-sky search either, see Figure 1. The position of the most signal-like cluster is $\alpha_{S}=46.49^{\circ} \delta_{S}=-64.97^{\circ}$ where five events are within one degree. Such amount of events is compatible with the background only hypothesis. The two-point auto-correlation function of neutrino candidates has been calculated looking for an excess at any level of angular separation. No significant excess of events has been found at any angular scale. 

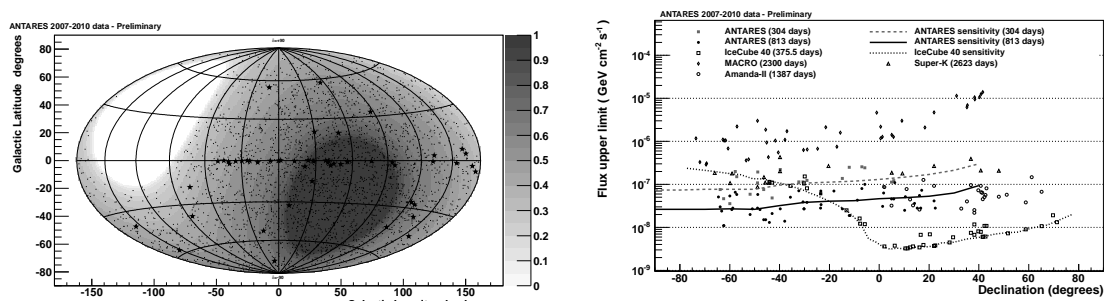

Fig. 1. Map in galactic coordinates of the 3058 selected neutrino candidates (dots) on the left. The positions of the 50 source candidates are indicated by the stars. Neutrino flux upper limits for an $E_{\nu}^{-2}$ spectrum from selected candidate sources on the right. The points show the $90 \%$ C.L. limit at the declination of the candidate source. Several previously published limits on source candidates are shown for comparison.

\section{Conclusions}

No significant excess of events has been found either with the full sky search or the candidate list search. Limits on the high energy neutrino flux have been calculated for a number of selected source candidates. The limits obtained for many of the candidate sources at declination below $-20^{\circ}$ are the most stringent to date.

\section{Acknowledgments}

The author gratefully acknowledges the financial support of the Spanish Ministerio de Ciencia e Innovación (MICINN), grants FPA2009-13983-C02-01, ACI2009-1020 and Consolider MultiDarkCSD2009-00064 and of the Generalitat Valenciana, Prometeo/2009/026.

\section{References}

2. J. A. Hinton and W. Hofmann, Annu. Rev. Astron. Astrophys. 47, 523 (2009).

3. E. Waxman and J. Bahcal, Phys. Rev. D 59, 023002 (1998).

4. M. Ageron et al. (ANTARES Collab.), Nucl. Instr. Meth. A656, 11 (2011).

5. P. Amram et al. (ANTARES Collab.), Nucl. Instr. Meth. A484, 369 (2002).

6. J. A. Aguilar et al. (ANTARES Collab.), Nucl. Instr. Meth. A622, 59 (2010).

7. J. A. Aguilar et al. (ANTARES Collab.), Astropart. Phys. 34, 652 (2011).

8. J. A. Aguilar et al. (ANTARES Collab.), Astropart. Phys. 34, 539 (2011).

9. S. Adrian-Martinez et al., to appear in Astrophys. J. Lett. [arXiv:1108.0292v1]. 